\title{
Research on Extraction of QAR Key Parameters during Approach Phase of Civil Aviation
}

\author{
Jianjun Chen ${ }^{\mathrm{a}}$, Xu Zhang ${ }^{\mathrm{b}}$, Ming Zhao ${ }^{\mathrm{c}}$, Yan Xia $^{\mathrm{d}}$ \\ College of Air Transportation, Shanghai University of Engineering Science, \\ Shanghai 201620, China. \\ achen8023miss@163.com, bzx_sues@163.com \\ cjmmolly_zhao@163.com, d18016002245@163.com
}

Keywords: Approach phase, QAR, R-type cluster analysis, flight quality.

\begin{abstract}
During the approach phase, the aircraft is prone to human error caused by the occurrence of unstable approach. In order to facilitate the study of the flight quality of the approach phase, this paper proposes to study on extraction of QAR key parameters in approach phase. The QAR parameters are classified by R-type clustering analysis, combined with the flight principle, the aircraft performance-related knowledge and the pilot's personal flight experience to judge. The variables within the group with higher correlation coefficients are reduced after each cluster. Ultimately, the main parameters of the QAR are the barometric altitude, the total air temperature, the wind speed, the wind direction, the pitch angle, the roll angle, the yaw angle, the indicated airspeed, the vertical speed, the longitudinal acceleration, the lateral acceleration and the vertical acceleration. Through these QAR key parameters, it can describe the trajectory and trend of the aircraft in the 3D space, which has laid a good foundation for studying the fight quality in approach phase.
\end{abstract}

\section{Introduction}

According to ICAO statistics, 80\% of flight accidents occurred in the aircraft takeoff and landing phase. Throughout these accidents and errors in the landing process, there is a common characteristic, that is, it begins with an unnatural and unstable approach. Once lost a stable approach, also lost the foundation of a safe landing. Quick access recorder (QAR) is used to monitor and record flight data acquisition of airborne equipment, covering most of the flight operation quality parameters. With the increase in the amount of recorded data, its complex structure, more properties for data analysis has brought many difficulties. Domestic and foreign scholars in the QAR data analysis, around the data feature extraction and classification of a lot of research. For example, Xiaorong Feng proposed the KPCA method based on EROS to deal with the similarity between QAR data ${ }^{[1]}$. Bin Gu uses the maximum margin principle to extract QAR data features ${ }^{[2]}$. Tsang gave the method of realizing large sample data by using the minimum closed ball technique with central constraint for the maximum interval discriminant analysis method ${ }^{[3]}$. Kim proposed an improved algorithm for QAR data logging and decoding ${ }^{[4]}$. However, there are few studies on the extraction of the essential parameters of flight quality from the recorded QAR parameters, and this kind of research is relatively lagging behind. For this reason, this paper selects QAR data of a company's flight to take the approach phase as the object of study, launches the analysis and research of the QAR data parameters, and forms the key parameter set of the aircraft approach phase.

\section{Selection of the Parameters}

QAR data in a complete record of the whole process of aircraft flight, it not only includes the historical manipulation of pilots, but also includes the meteorological environment, flight attitude and other information.Referring to the A321 aircraft performance manual, the QAR parameters affecting the flight approach are initially selected. As shown in Table 1 below. If all of these is used to establish the model to study the flight quality of the approach phase, meanwhile, because of the interaction 
between the parameters, the stability of the results will also be affected. Therefore, before we establish the model to study the flight quality, it is necessary to reduce the parameters, extract the QAR key parameters in the approach phase, keep the parameters with large influence, and ignore the small parameters. Of course, follow-up to the actual study of specific flight safety, but also according to the need to add other parameters.

Table 1 Variable of approach phase

\begin{tabular}{ccc}
\hline & Parameter name \\
\hline 1. Barometric altitude & 2. Total air temperature & 3. Wind speed \\
4. Wind direction & 5. Pitch angle & 6. Roll angle \\
7. Yaw angle & 8. Attack angle & 9. Indicated airspeed \\
10. True airspeed & 11. Ground speed & 12. Vertical speed \\
13. Mach number & 14. Lateral acceleration & 15. Longitudinal acceleration \\
16. Vertical acceleration & 17. Radio height & 18. N1 actual engine 1 \\
\hline
\end{tabular}

\section{Standardization of Data}

QAR data comes from an airline A321 type aircraft, intercepted radio height at 2000 feet away from the airport to the main air ground switch grounding's 251 data to study on extraction of QAR key parameters during approach phase. Since the variables exist between different dimensional and dimensional units, such a situation affects the clustering results of variables. In order to eliminate the influence of dimension on the relationship between variables, first, the sampling rate of the variables is unified, and then standardized processing is performed.Since the analysis done here is not an overrun analysis, only the relationship between the trends of the variables in the aircraft approach phase is sought. So we can unify the sampling rate by finding the mean of the variable per second ${ }^{[12]}$. Standardized processing uses Z-score standardization, which is the most commonly used standardized method in SPSS.

\section{Classification and Reduction of QAR Parameters}

\subsection{The Concept of Clustering Analysis.}

Cluster analysis is a multivariate statistical method to study the classification of samples or variables. Compared with other classification methods, clustering analysis can be realized quickly through software such as SPSS. According to the analysis object can be divided into R-type clustering and Q-type clustering, R-type clustering is the classification of variables, Q-type clustering is the classification of samples. In the classification of QAR data variables, the use of R-type clustering, separation of changes in the trend of large differences in variables, small differences will be gathered together ${ }^{[5]}$.

\subsection{Cluster Analysis and Parameter Reduction.}

Among cluster analysis, in order to determine the relationship between the variables, we need to calculate the correlation coefficient between the data, R-type cluster analysis using the standard is Pearson correlation, the correlation coefficient of the variables are first clustered into a class. The correlation coefficient is calculated as follows.

$$
r=\frac{\sum(x-\bar{x})(y-\bar{y})}{\sqrt{\sum(x-\bar{x})^{2}} \cdot \sqrt{\sum(y-\bar{y})^{2}}}
$$

The absolute value of the correlation coefficient $\mathrm{r}$ is closer to 1 or minus 1 , and the correlation is stronger, the closer it is to 0 , and the correlation is weaker.

As the number of variables is not reduced after clustering analysis, the parameter reduction is also needed. Although the parameter reduction can be used to reduce the dimension of the data. But only from the data changes in the law to achieve this process is easy to ignore the impact of variables on 
the actual flight. The relationship between variables is not only reflected in the numerical, the change of different variables can sometimes lead to the same insecurity, as the trend is similar, leading to similar or even overlap in describing the motion of an aircraft.Therefore, on the basis of cluster analysis,combined with the flight principle, the aircraft performance-related knowledge and the pilot's personal flight experience to judge, only the dominant variables are retained, other variables are ignored, and the variables are explained and reduced. As shown in Figure 1, the dendrogram of the selected parameter variable is first analyzed by the R cluster analysis.

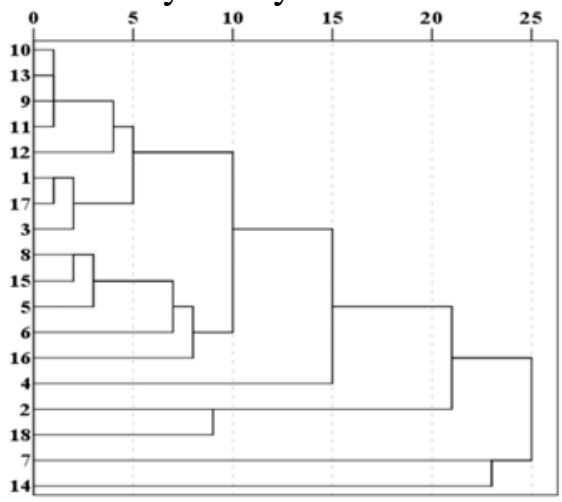

Fig. 1 Dendrogram of the first cluster analysis

The clustering process of several variables with correlation coefficients more than 0.99 is shown in Table 2 below.

Table 2 Process of first clustering

\begin{tabular}{ccccc}
\hline step & number of cluster & correlation coefficient & \multicolumn{2}{c}{ merged variables } \\
\hline 1 & 17 & 1.000 & 10 & 13 \\
2 & 16 & 1.000 & 1 & 17 \\
3 & 15 & 0.999 & 9 & 10 \\
4 & 14 & 0.996 & 9 & 11 \\
\hline
\end{tabular}

The results showed that at the level of correlation coefficient greater than 0.99 , variable 9 is combined with variables 10, 11 and 13 into a set, and variables 1 and 17 are combined into one set.

In the approach phase, the indicated airspeed can accurately reflect the dynamic relationship between the air and the airplane, which is a very important parameter of the flight control. In the flight quality monitoring work, the speed of the monitoring is usually also taken to indicate airspeed as a monitoring indicator. The true airspeed can be calculated according to the indicated airspeed, barometric altitude and air temperature, and can be estimated by using mental arithmetic when the airspeed is indicated. When the wind speed and wind direction are known, according to the speed of travel triangles, using the foot algorithm and the heart algorithm can calculate the ground speed. Low and low speed flight, Mach number as the ratio of true airspeed and sound speed, not very large, then the ratio does not have great significance. Therefore, variable 9 is associated with variables 10, 11, and 13, finally, we reserve the variable 9 . The height of flight is measured by radio height altimeter and barometric altitude altimeter, flight height is usually calculated based on the barometric at which altitude is measured, radio height can be measured by the propagation and reflection characteristics of radio waves. By comparison, the barometric altitude contains more information and has higher impact factors, so variables 1 and 17 retain the variable 1 .

The second cluster is also clustered into a class according to the variables with higher correlation coefficients. The dendrogram of the cluster analysis is shown in Figure 2 below. 


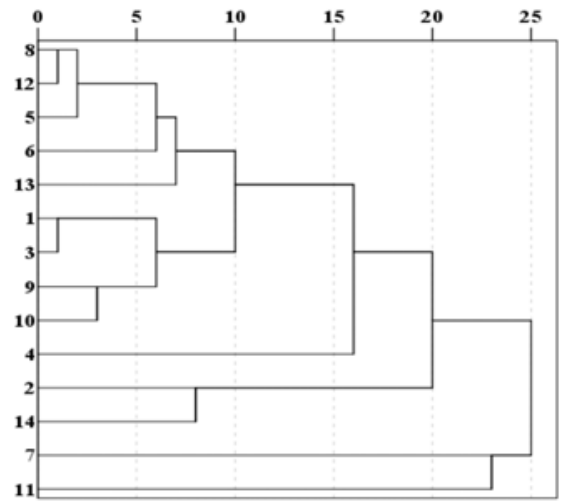

Fig. 2 Dendrogram of the second cluster analysis

The clustering process of the second cluster correlation coefficient more than 0.85 is shown in Table 3 below.

Table 3 Process of first clustering

\begin{tabular}{ccccc}
\hline step & number of cluster & correlation coefficient & \multicolumn{2}{c}{ merged variables } \\
\hline 1 & 13 & 0.994 & 8 & 12 \\
2 & 12 & 0.932 & 1 & 3 \\
3 & 11 & 0.859 & 5 & 8 \\
4 & 10 & 0.851 & 9 & 10 \\
\hline
\end{tabular}

After the second clustering, variables 5, 8, and 12 are merged into a set. Pitch angle can not be eliminated as the key parameter to reflect the attitude of aircraft. The longitudinal acceleration is that the acceleration produced by the thrust of the engine and the resistance of the aircraft on the vertical axis. It contains more information and can not be eliminated. As the aerodynamic performance parameters of the attack angle, the aircraft in the approach phase is not stalled, the flight quality is not considered as a key parameter, so the variables 5, 8, 12 are retained 5 and 12. Variables 1 and 3 are important meteorological environment parameters of flight. The effect of barometric altitude on the aircraft is actually the effect of air density on the aircraft. Sudden changes in wind speed in horizontal or vertical directions can cause abrupt changes in flight height and flight attitude, which can cause wind shear. In approaching phase, these two variables can not be neglected, so variables 1 and 3 are retained. The variables 9 and 10 are clustered together, and the correlation is very large. But the rate of descent should be taken into account in the process of approaching, and the vertical velocity as a token of the drop rate, has great influence on the flight quality during the actual flight, there is no reduction here. Variable 14, as the thrust of the engine, is only weakly correlated with the total temperature of the atmosphere. It is suitable for the study of engine performance and fuel consumption. It is not used as a parameter to study the flight quality.

After second reductions, the key parameter of QAR in the approach phase of civil aviation is obtained. The key parameters of the QAR are the barometric altitude, the total air temperature, the wind speed, the wind direction, the pitch angle, the roll angle, the yaw angle, the indicated airspeed, the vertical speed, the longitudinal acceleration, the lateral acceleration and the vertical acceleration.

\section{Summary}

In this paper, R-clustering method is used to get the key parameters of QAR in the approach phase. Through these variables can describe the trajectory and trend of the aircraft in the 3D space, which has laid a good foundation for studying the fight quality in approach phase.

\section{References}

[1]. Feng Xiao-rong, Feng Xing-jie, Feng Zeng-cai. Similarity analysis of QAR data sets based on K-EROS [J]. Computer Engineering and Application, Vol. 48 (2012) No. 9, p. 108-110.

[2]. Gu Bin, Wang Jian-dong. A novel feature extraction method for QAR data [J]. Journal of Sichuan University (Engineering Science Edition), Vol. 43 (2011) No. 3, p. 113-117. 
[3]. Tsang I W, Kocsor A, Kwok J T. Efficient kernel feature extraction for massive data sets[C]//Proceedings of the 12th ACM SIGKDD international conference on Knowledge discovery and data mining. ACM, 2006, p. 724-729.

[4]. Kim J H, Lyou J. Enhanced QAR Flight Data Encoding and Decoding Algorithm for Civil Aircraft[C]// Sice-Icase International Joint Conference. IEEE, 2006, p. 5169-5173.

[5]. Sun Rui-shan,Yang Yi-xuan. Research on Extraction of Key Parameters during Take-off Based on QAR Data [J]. Comprehensive transportation, Vol. 37 (2015) No. 9, p. 9-11. 